\title{
Is endoscopic band ligation the gold standard for gastric antral vascular ectasia?
}

\section{다)(요 $\odot$}

\author{
Authors \\ Marcel Tantau ${ }^{1,2}$, Dana Crisan ${ }^{1}$ \\ Institutions \\ 1 "Iuliu Hatieganu" University of Medicine and Pharmacy, \\ Cluj-Napoca, Romania \\ 2 “Octavian Fodor" Regional Institute of Gastroenterology \\ and Hepatology, Cluj-Napoca, Romania
}

\author{
Bibliography \\ DOI https://doi.org/10.1055/a-1006-2763 | \\ Endoscopy International Open 2019; 07: E1630-E1631 \\ (c) Georg Thieme Verlag KG Stuttgart · New York \\ eISSN 2196-9736 \\ Corresponding author \\ Marcel Tantau, Iuliu Hatieganu University of Medicine and \\ Pharmacy, Faculty of Medicine, Cluj Napoca, Romania \\ matantau@gmail.com
}

The endoscopic treatment of gastric antral vascular ectasia (GAVE) is a relatively uncharted field with few reliable techniques available for the time being.

As is well known, assessed from a histological standpoint, GAVE consists of dilated and tortuous capillaries in the mucosal layer, often associated with intraluminal thrombi and accompanied by dilated and tortuous submucosal veins, without significant inflammation. The typical location of these capillaries is in the gastric antrum, with variations in overall shape and extent. The exact pathophysiological pathway is relatively unknown. Several hypotheses have been proposed, including mechanical stress due to the relatively higher pressure in the antrum, hormone imbalance mostly related to hypergastrinemic states, and an association with tyrosine kinase inhibitors. [1-3] Yet none of the above appears to have any direct implication regarding our current therapeutic options. However, the association with chronic kidney disease, advanced liver disease, systemic sclerosis, and metabolic syndrome is unquestionable, suggesting that optimizing management of these chronic diseases should improve the outcome of secondary conditions such as GAVE.

As we currently understand the problem, it appears that we have hit a deadlock regarding our treatment options, as the available methods are few and far from perfect.

No pharmacological treatment has proven to be effective in this setting, with only modest endeavors published in this field. The lack of a proper understanding of the pathophysiology might be a feasible explanation. Estrogen-progesterone regimens for cirrhotic patients with GAVE [4] were tried more than 20 years ago, but the data were not strong enough for validation and no significant subsequent studies were performed. An association between proton pump inhibitors (PPIs) and
GAVE has also been proposed (acid suppression leading to hypergastrinemia) $[5,6]$, but discontinuing PPIs can hardly be called a therapeutic option.

Surgery is a delicate option in GAVE, because most patients carry the burden of multiple and severe comorbidities. Unfortunately, the risk/benefit analysis can often be tilted towards undesirable events, and operating remains a viable option only for emergency refractory cases.

Therefore, the only viable solutions we have for GAVE are concentrated in the field of endoscopic therapy. The array of available options can be divided into two groups, based on layer penetration: superficial (action limited to the mucosa) and deep (including submucosa). Superficial techniques act mostly on the mucosal layer and are represented by argon plasma coagulation (APC) and radiofrequency ablation (RFA). Cryotherapy and Nd:YAG laser therapy have been largely abandoned due to relative ineffectiveness, high complication rates, and availability issues. The only deep submucosal technique available is endoscopic band ligation (EBL).

Judging by the histological appearance of GAVE, with dilated and tortuous submucosal veins, along with extensive mucosal lesions, one could empirically imply that reaching a more profound layer should generate better outcomes. However, APC has long been the staple of GAVE treatment. APC generates good immediate results, with therapeutic success ranging between $70 \%$ and $90 \%$, although recurrent bleeding and further interventions were frequently required, ranging from a reasonable $35 \%$ to an inefficient $78.9 \%$ [7-11]. Only recently has the tide appeared to be shifting towards EBL, after first being used as salvage therapy in a case of APC refractory GAVE bleeding [12]. Since then, multiple papers have been published on EBL describing relatively favorable outcomes. One retrospective 
comparison between EBL and APC showed a significantly lower recurrent bleeding rate in favor of EBL (8\% vs. 68\%) [13]. Another similar study showed that therapeutic success after EBL was obtained in $100 \%$ of cases, compared to $48 \%$ for APC [14]. A prospective comparative study in a pediatric population also hinted at the superiority of EBL, requiring fewer and shorter sessions and a significantly decreased need for blood transfusions [15]. Up to this point, there is only one randomized controlled study of EBL and APC in treatment of gastric antral and fundal vascular ectasia in cirrhotic patients, suggesting the superiority of EBL in terms of number of sessions and blood transfusion units needed [16]. Other previous reports, including a previous analysis performed by the same group, followed the same trend $[17,18]$. The current article published by Eccles et al. [19] regarding long-term follow-up of patients with GAVE treated with EBL, further strengthens the case for expanding use of EBL in GAVE, with high initial success rate and relatively low recurrent bleeding rates, albeit in a small sample.

There are several theoretical advantages of EBL, when compared to the other endoscopic techniques, which of course need further validation. Per se, EBL is cheaper than APC, and it is widely available. It also appears that fewer procedures are needed, therefore further reducing costs. Also, long-term fibrosis in the submucosa should, at least theoretically, reduce reoccurrence rates by preventing dilatation of new small venous tracts. Not least, EBL requires less experience, and can be performed by less experienced endoscopists, with instant confirmation of procedure success, which can, as a consequence, expand the availability of the method.

The trend of recent research, strongly enforced by the current article, combined with the inherent and theoretical advantages of EBL is promising. However, do we have enough data to proclaim the supremacy of EBL in GAVE? There are only a few prospective studies comparing APC and EBL, but the results favor the latter. Expanding on the current dataset could give rise to a new gold standard in the field, sooner rather than later.

\section{Competing interests}

None

\section{References}

[1] Zepeda-Gómez S. Endoscopic treatment for gastric antral vascular ectasia: current options. GE Port J Gastroenterol 2017; 24: 176-182

[2] Jabbari M, Cherry R, Lough JO et al. Gastric antral vascular ectasia: the watermelon stomach. Gastroenterol 1984; 87: 1165-1170

[3] Smith E, Davis ], Caldwell S. Gastric Antral Vascular Ectasia Pathogenesis and the Link to the Metabolic Syndrome. Curr Gastroenterol Rep 2018; 20: 36
[4] Tran A, Villeneuve JP, Bilodeau M et al. Treatment of chronic bleeding from gastric antral vascular ectasia (GAVE) with estrogen-progesterone in cirrhotic patients: an open pilot study. Am J Gastroenterol 1999; 94: 2909-2911

[5] Nakade Y, Ozeki T, Kanamori $\mathrm{H}$ et al. A case of gastric antral vascular ectasia which was aggravated by acid reducer. Case Rep Gastroenterol 2017; 11: 64-71

[6] Parkman HP, Urbain JL, Knight LC et al. Effect of gastric acid suppressants on human gastric motility. Gut 1998; 42: 243-250

[7] Chiu YC, Lu LS, Wu KL et al. Comparison of argon plasma coagulation in management of upper gastrointestinal angiodysplasia and gastric antral vascular ectasia hemorrhage. BMC Gastroenterol 2012; 12: 67

[8] Chaves D, Sakai P, Oliveira CV et al. Watermelon stomach: clinical aspects and treatment with argon plasma coagulation. Arq Gastroenterol 2006; 43: 191 - 195

[9] Lecleire S, Ben-Soussan E, Antonietti M et al. Bleeding gastric vascular ectasia treated by argon plasma coagulation: a comparison between patients with and without cirrhosis. Gastrointest Endosc 2008; 67: $219-225$

[10] Herrera S, Bordas JM, Llach J et al. The beneficial effects of argon plasma coagulation in the management of different types of gastric vascular ectasia lesions in patients admitted for $\mathrm{Gl}$ hemorrhage. Gastrointest Endosc 2008; 68: 440-446

[11] Fuccio L, Zagari RM, Serrani M et al. Endoscopic argon plasma coagulation for the treatment of gastric antral vascular ectasia-related bleeding in patients with liver cirrhosis. Digestion 2009; 79: 143-150

[12] Kumar R, Mohindra S, Pruthi HS. Endoscopic band ligation: a novel therapy for bleeding gastric antral vascular ectasia. Endoscopy 2007; 39: E56-E57

[13] Sato T, Yamazaki K, Akaike J. Endoscopic band ligation versus argon plasma coagulation for gastric antral vascular ectasia associated with liver diseases. Dig Endosc 2012; 24: 237-242

[14] Keohane J, Berro W, Harewood GC et al. Band ligation of gastric antral vascular ectasia is a safe and effective endoscopic treatment. Dig Endosc 2013; 25: $392-396$

[15] Ghobrial C, Rabea M, Mohsen N et al. Gastric antral vascular ectasia in portal hypertensive children: Endoscopic band ligation versus argon plasma coagulation. J Pediatr Surg 2019; 54: 1691-1695

[16] Elhendawy M, Mosaad S, Alkhalawany W et al. Randomized controlled study of endoscopic band ligation and argon plasma coagulation in the treatment of gastric antral and fundal vascular ectasia. United Europ Gastroenterol J 2016; 4: 423-428

[17] Zepeda-Gomez S, Sultanian R, Teshima CW et al. Gastric antral vascular ectasia: a prospective study of treatment with endoscopic band ligation. Endoscopy 2015; 47: 538-540

[18] St Romain P, Boyd A, Zheng J et al. Radiofrequency ablation (RFA) vs. argon plasma coagulation (APC) for the management of gastric antral vascular ectasia (GAVE) in patients with and without cirrhosis: results from a retrospective analysis of a large cohort of patients treated at a single center. Endosc Int Open 2018; 06: E266-E270

[19] Eccles J, Falk V, Montano-Loza A] et al. Long-term follow-up in patients with gastric antral vascular ectasia (GAVE) after treatment with endoscopic band ligation (EBL). Endosc Int Open 2019; 07: E16240 E1629 